\title{
The contribution of the lexical component in hybrid clustering, the case of four decades of "Scientometrics"
}

\author{
Bart Thijs $^{1} \cdot$ Wolfgang Glänzel ${ }^{2,3}$
}

Received: 8 January 2018/Published online: 2 February 2018

(C) Akadémiai Kiadó, Budapest, Hungary 2018

\begin{abstract}
The introduction of textual analysis and the use of lexical similarities already proved an important asset in science mapping. Earlier research showed the added value of hybrid document networks over link-based ones through the reduction of the extreme sparseness. However, it was only after the application of Natural Language Processing and phrase extraction that networks purely based on lexical similarities could be used as input for topic detection in quantitative science studies. This study investigates the contribution of the lexical component in hybrid cluster on a set of articles published in the journal Scientometrics since its foundation during four decades. Shifting the weight of the lexical components generates changes in the structure of the underlying hybrid network, which can be detected through clustering techniques. We show that these changes are not moving documents randomly, but in fact identify small groups of papers either at the borderline between different topics or combining those. In addition, the analysis substantiates that the lexical component adopts the structure of the network rather than amplifies hidden structures of the link-based network.
\end{abstract}

Keywords Science mapping $\cdot$ Lexical similarity $\cdot$ Hybrid clustering $\cdot$ Natural Language Processing · Scientometrics

Bart Thijs

bart.thijs@kuleuven.be

Wolfgang Glänzel

wolfgang.glanzel@kuleuven.be; glanzw@iif.hu

1 ECOOM, KU Leuven, Louvain, Belgium

2 ECOOM and Department of MSI, KU Leuven, Louvain, Belgium

3 Department of Science Policy and Scientometrics, Library of the Hungarian Academy of Sciences, Budapest, Hungary 


\section{Introduction}

Eugene Garfield, one of the first Editors-in-Chief of the journal Scientometrics, introduced the Permuterm Subject Index (PSI) of the Science Citation Index database as early as in the year 1969 (see Garfield 1969). At that time, PSI went far beyond a conventional title-word index. The PSI listed under each term all the other title words with which it has appeared. The refinement enabled the user to search a combination of two terms, thus increasing the specificity of the search and decreasing the percentage of irrelevant material. Nonetheless, it was more than twenty years later that textual analysis and the use of lexical similarities have been incorporated in the context of science mapping where co-citation analysis was in the foreground. This late entrance was mainly due to the lack of electronically available databases holding textual information like abstracts or even full text. But, also the processing resources needed for these analyses were not at hand for most of the researchers in quantitative science studies. Co-word analysis based on the co-occurrence of keywords or other controlled terms was one of the first techniques applied (Callon et al. 1991) along with co-heading analysis (Todorov and Winterhager 1990). Later, Glenisson et al. (2005) started using full texts for an improved interpretation of the link-based structure of document networks.

The use of truly hybrid techniques was proposed to overcome the shortcoming of linkbased techniques (Janssens et al. 2008) but also to reduce the fuzziness and noise of keyword- and term-based techniques. In that approach, the links based on bibliographic coupling and lexical similarities were combined into new 'hybrid' links or edges in the document network prior to the analysis. Bibliographic coupling and other citation-link based approaches are notoriously prone to false negatives (Glänzel and Thijs 2012). The advantages of hybrid techniques have been confirmed by other authors (e.g., Boyack and Klavans 2010). In the attempt for a hybrid approach, Janssens et al. opted for a combination of the two components using a highly complex method which calculated for each weighted link the probability that it would occur in a completely random network with the same properties (number of nodes, edges) as the original document network. This approach could cope with the differences in distribution of the weights of the links in both networks but was too complex to be calculated for large networks. We took a more pragmatic approach (Glänzel and Thijs 2011) by using a weighted linear combination of the angles between document pairs in the assumed Vector Space Model resulting in hybrid cosine similarities the calculation of which proved quite straightforward. One issue still remained when using single terms representing the lexical component, particularly, the high dimensionality with fussiness and the proneness to produce false positives. This limited the applicable weight of the lexical component of the hybrid approach to an inacceptable extent, notably in the social sciences and the humanities, where citations play a less significant part as in the natural sciences. The problem related to the distributional properties were only solved after the introduction of noun phrase extraction through the application of Natural Language Processing (Thijs et al. 2017).

After the introduction of NLP, the constraints on the weighting factor for both components in the hybrid combination was released. When using all terms found in a document, the network tended to be very dense, which resulted in practically useless network bases for cluster analysis. The extraction of noun phases and removal of single terms from the term vectors allowed to create much sparser networks and obtain valid cluster solutions solely based on lexical similarities (Thijs et al. 2017). These properties were further explored and confirmed in the analysis of the astronomy dataset (Glänzel and Thijs 2017), where clusters from a pure bibliographic-coupling network were compared with two hybrid 
networks, one using all terms with a $75 \%$ weight on the citation-link based component and one built after noun phrase extraction with a $75 \%$ weight on the lexical component.

After we could demonstrate the validity and the benefits of the use of noun phrase extraction for the clustering of hybrid network we would like to extend our understanding of the role or contribution of the lexical component in hybrid clustering. This brings us to the objective of the present study, which is to investigate in a more qualitative manner the added value of the lexical component in hybrid clustering and analyse how it influences the outcome of different cluster solutions. We will use the weighting parameter in the hybrid combination as some kind of shifter, which allows us to detect minor changes in the network and cluster structure. The focus lies on the following two main research questions.

1. Are there differences that can be observed after adding more weight to the lexical component valid?

2. And, if so, can these changes only be attributed to the inclusion of the lexical information or is it already hidden in the citation-link based network?

The paper proceeds as follows. After the description of the dataset and the applied methodology for processing and clustering, we will present the cluster solutions of a pure bibliographic coupling network and a pure lexical one. Then we look at overlaps or concordance between these cluster solutions and try to identify similar and different clusters. By changing the component weight in the combination, we will generate intermediate networks and look at the flow of documents between the detected clusters. This gives us insight into the validity of these changes. In a last analysis, mapping of one cluster structure on another network and the use of clustering analysis within a specific group are used to answer the second question which relates to the hidden presence of subtopics or groups of papers possibly representing latent structures.

\section{Data and methodology}

The study is based on all citable documents published in the journal Scientometrics from its very first issue in 1979 until December 2017. The bibliographic data from these papers was retrieved from Clarivate Analytics Web of Science combined with their cited references and abstracts. In addition, full texts were retrieved in PDF-format and parsed using the Science-Parse library developed by allenai.org and provided through github (https:// github.com/allenai/science-parse). This parser is able to extract different sections in scientific papers and label these according to the section titles provided in the full text. This allows us to distinguish between the canonical sections in the paper like 'Introduction', 'Data and Methodology', 'Results' and 'Discussion and Conclusions'.

Noun-phrases extraction was applied both on abstracts and full text of each paper using the Stanford Core NLP package (Manning et al. 2014). The presence of the section headers adds another dimensionality to the characterisation of the extracted noun phrases.

Lexical similarities between documents are calculated as the cosine between the TFiDF vectors representing the documents. Cited references found in each document are the basis for the bibliographic coupling using cosine similarity.

Document networks are built using either the lexical or the bibliographic-coupling similarities but also hybrid networks where the hybrid similarities $r$ are defined as the cosine of the linear combination of the underlying angles between the vectors representing the corresponding documents in the vector space model, i.e., 


$$
r=\cos (\lambda \cdot \arccos (\eta)+(1-\lambda) \cdot \arccos (\xi)), \quad \lambda \in[0,1],
$$

where $\eta$ is the similarity defined on bibliographic coupling and $\xi$ the textual similarity. The $\lambda$ parameter defines the convex combination, $\arccos (\eta)$ and $\arccos (\xi)$, respectively, denoting the two underlying angles and takes one of these values $0.25,0.5$ and 0.75 . Actually, the pure bibliographic coupling and lexical networks can be obtained by choosing 0 or 1 , respectively, as weighting parameter. For each network, we used a similarity threshold of 0.025 . The igraph implementation of the Louvain community detection algorithm (Blondel et al. 2008) was used for clustering the documents in the largest component and only the partitioning at the highest level was retained. Clusters were characterized by both the noun phrases from the abstract and from the full text with the highest TFiDF and the most cited references.

\section{Results}

\section{Bibliographic coupling network}

The largest component of the network comprises 3964 documents with a density of $4.09 \%$. The clustering resulted in eight clusters with a modularity of 0.479 . The labels, which are obtained from the most significant noun phrases, and the sizes of each cluster are given in Table 1. The table also lists the noun phrases from the introduction section of the papers with the highest sum of weighted TFiDF values. These are obtained from the TFiDF values of the noun phrases divided by the norm of the vector representing the document. Each cluster is also coded by a letter which will be used for linking each solution. The subscript index of the codes refers to the weight of the lexical component in this approach. In Table 1 pure citation-link based results are given, hence the zero weight and the index 0 .

Table 1 Code, size, label and noun phrases taken from the introduction of papers from eight clusters in the bibliographic-coupling network of all papers in Scientometrics. Data sourced from Clarivate Analytics Web of Science Core Collection

\begin{tabular}{|c|c|c|c|}
\hline Cluster code & Size & Label & Noun phrases in introduction \\
\hline$A_{0}$ & 465 & Patents \& Technology & $\begin{array}{l}\text { Patent Citation } \\
\text { Patent Application }\end{array}$ \\
\hline$B_{0}$ & 923 & Collaboration & $\begin{array}{l}\text { International Collaboration } \\
\text { Co-Authorship }\end{array}$ \\
\hline$D_{0}$ & 880 & Journal Impact Measures & $\begin{array}{l}\text { Journal Impact Factor } \\
\text { Peer Review }\end{array}$ \\
\hline$G_{0}$ & 130 & Web-related Studies & $\begin{array}{l}\text { Research Trend } \\
\text { Bibliometric Methodology }\end{array}$ \\
\hline$H_{0}$ & 344 & Citation Analysis & $\begin{array}{l}\text { Power Law } \\
\text { Citation Distribution }\end{array}$ \\
\hline$I_{0}$ & 427 & Research Performance & $\begin{array}{l}\text { h-index } \\
\text { Google Scholar }\end{array}$ \\
\hline$J_{0}$ & 128 & Institutional Performance & $\begin{array}{l}\text { Higher Educ } \\
\text { University Ranking }\end{array}$ \\
\hline$K_{0}$ & 667 & Science Mapping & $\begin{array}{l}\text { Co-Citation Network } \\
\text { Co-Word Analysis }\end{array}$ \\
\hline
\end{tabular}


Beyond distinguishing these eight topics covered by the journal Scientometrics we can also add the publication date as a further dimension of the analysis. In Fig. 1, we use a modified Activity Index to present the evolution of the share of each cluster over 5 publication windows. The share of each cluster within the distinct windows are gauged against the share of the cluster in the complete document set. This approach provides a balanced image along both axes, particularly, within each time period and each cluster.

The boundaries of these time windows are chosen to create publication sets of approximately equal size instead of similar length, hence the long first window of 14 years and two closing windows of 3 years each. Despite the different time lengths, we see a clear evolution in the partitioning of the publication set. In the first period, Citation Analysis with a strong focus on the study of different distributions was predominant over other topics such as Collaboration and Science Mapping. As mentioned in the introduction, the main focus within this last topic was the use of co-citation analysis. The attention for this topic has gradually decreased. But after 2010, the decline was stopped, and new researchers have again found interest in this topic resulting in the introduction of global clustering solutions, hybrid approaches and visualization techniques. Regarding the cluster on Research Performance the noun phrase with the highest TFiDF suffices to explain the evolution. The publication of Hirsch's paper initiated a series of articles studying the properties of the h-index and proposing derivatives and solution for improvement. Of course, all these papers refer to the seminal paper and thus create many bibliographiccoupling links. The same phenomenon, but to a lesser extend can be observed related to institutional ranking with the conception of the Academic Ranking of World Universities

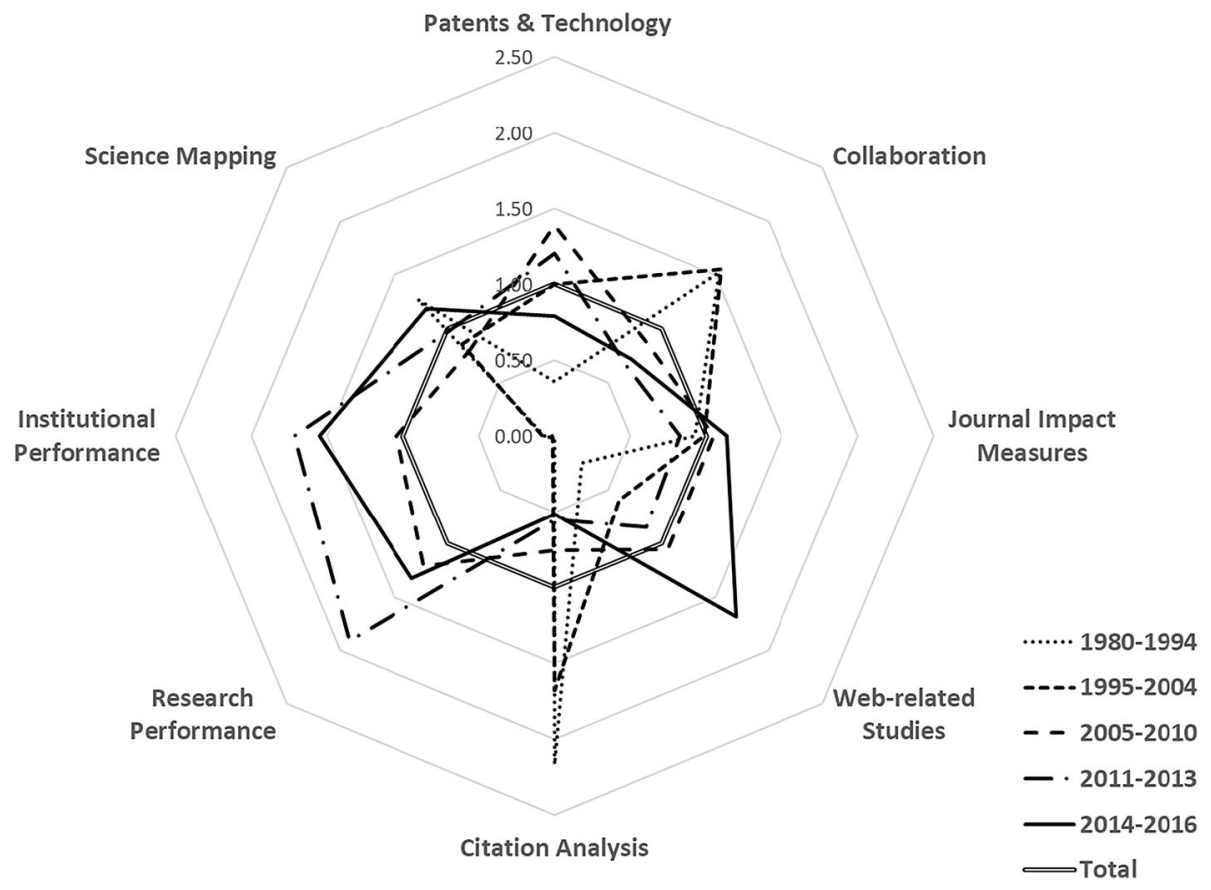

Fig. 1 Evolution of the share of 8 clusters in a bibliographic-coupling network of papers published in the journal Scientometrics between 1979 and 2016. Data sourced from Clarivate Analytics Web of Science Core Collection 
(ARWU; http://www.shanghairanking.com/) and other university rankings like the Times Higher Education World University Ranking (THES; https://www.timeshighereducation. com/world-university-rankings) and the CWTS Leiden Ranking (http://www.leidenranking. com/). Apparently, the only stable cluster over time can be partially attributed to Eugene Garfield and comprises studies on journal impact measures with Journal Impact Factor as being the most important noun phrase. None of these observations account for any surprise when comparing to previous studies mapping the field, however this is not the objective of the study at hand.

\section{Lexical Similarities}

In total, 4376 publications are clustered resulting in a set of 10 different partitions. The difference in clustered documents between both networks is caused by the restriction of the analysis to the largest component. The bibliographic-coupling network is less dense and contains more singletons or unconnected pairs or triplets. Including those unconnected nodes in the clustering has a negative effect on the interpretability of the different scores.

Table 2 presents the 10 clusters with their code (the subscript index refers to $100 \%$ lexical approach), size, label and noun phrases. In-depth analysis of the clusters by looking at the titles of the documents, noun phrases from abstract, introduction and conclusions of papers proved the validity of the obtained clusters.

The evolution of the 10 clusters is given in Fig. 2 and shows a much smoother picture than the previous one based on bibliographic coupling (Fig. 1). We find a strong outlier for

Table 2 Code, size, label and noun phrases taken from the introduction of papers from ten clusters in the lexical similarity based network of all papers in Scientometrics. Data sourced from Clarivate Analytics Web of Science Core Collection

\begin{tabular}{|c|c|c|c|}
\hline Code & Size & Label & Noun phrases in introduction \\
\hline$A_{100}$ & 778 & Patents \& Technology & $\begin{array}{l}\text { Patent Citation } \\
\text { Patent Application }\end{array}$ \\
\hline$B_{100}$ & 177 & Impact Measures \& Metrics & $\begin{array}{l}\text { Citation Impact } \\
\text { Basic Research }\end{array}$ \\
\hline$C_{100}$ & 553 & Collaboration & $\begin{array}{l}\text { International Collaboration } \\
\text { Co-Authorship }\end{array}$ \\
\hline$D_{100}$ & 431 & Journal Impact Measures & $\begin{array}{l}\text { Journal Impact Factor } \\
\text { Journal Citation Report }\end{array}$ \\
\hline$F_{100}$ & 631 & Research Assessment & $\begin{array}{l}\text { Higher Education } \\
\text { Research Performance }\end{array}$ \\
\hline$G_{100}$ & 243 & Web-related Studies & $\begin{array}{l}\text { Peer Review } \\
\text { Google Scholar }\end{array}$ \\
\hline$H_{100}$ & 335 & Research Production & $\begin{array}{l}\text { Scientific Production } \\
\text { Research Group }\end{array}$ \\
\hline$I_{100}$ & 291 & Citation Analysis & $\begin{array}{l}\text { Citation Distribution } \\
\text { Highly Cited Paper }\end{array}$ \\
\hline$J_{100}$ & 477 & Field and Regional Studies & $\begin{array}{l}\text { Social Science } \\
\text { Research Trend }\end{array}$ \\
\hline$K_{100}$ & 460 & Science Mapping & $\begin{array}{l}\text { Co-Citation } \\
\text { Intellectual Structure }\end{array}$ \\
\hline
\end{tabular}


the first period (1979-1994) for the new cluster Impact Measures \& Metrics. The changes in the Web-related Studies are obvious.

\section{Concordance between bibliographic coupling and lexical clusters}

Looking at Tables 1 and 2 with cluster labels and noun phrases allows some preliminary observations on the concordance of the two approaches. The labels of seven out of eight clusters in the bibliographic coupling scenario can be found in the lexical approach. This indicates that the techniques used for the identification of the labels return similar results. Indeed, the most frequently occurring noun phrases combined with an inspection of the titles of most cited documents per cluster are quite similar between the two approaches for several clusters. This is also confirmed by the cosine similarities between the cluster solution as presented in Table 3 . The column headers contain the cluster code for the pure lexical approach hence the 100 as subscript index and the row header refers to the bibliographic coupling in which the lexical component has weight zero.

Two cells have a value above 0.5. Patents \& Technology and Science Mapping are found in both approaches and their evolution over time shows similar patterns. As mentioned above, the evolution in the lexical approach is smoother but the same pattern can be observed. The slight difference in dynamics indicate some changes between the two approaches. Another strong tie can be found between the clusters labelled as Collaboration and Journal Impact Measures. Here we see much stronger effects over time where we could see a high peek for collaboration in bibliographic coupling.

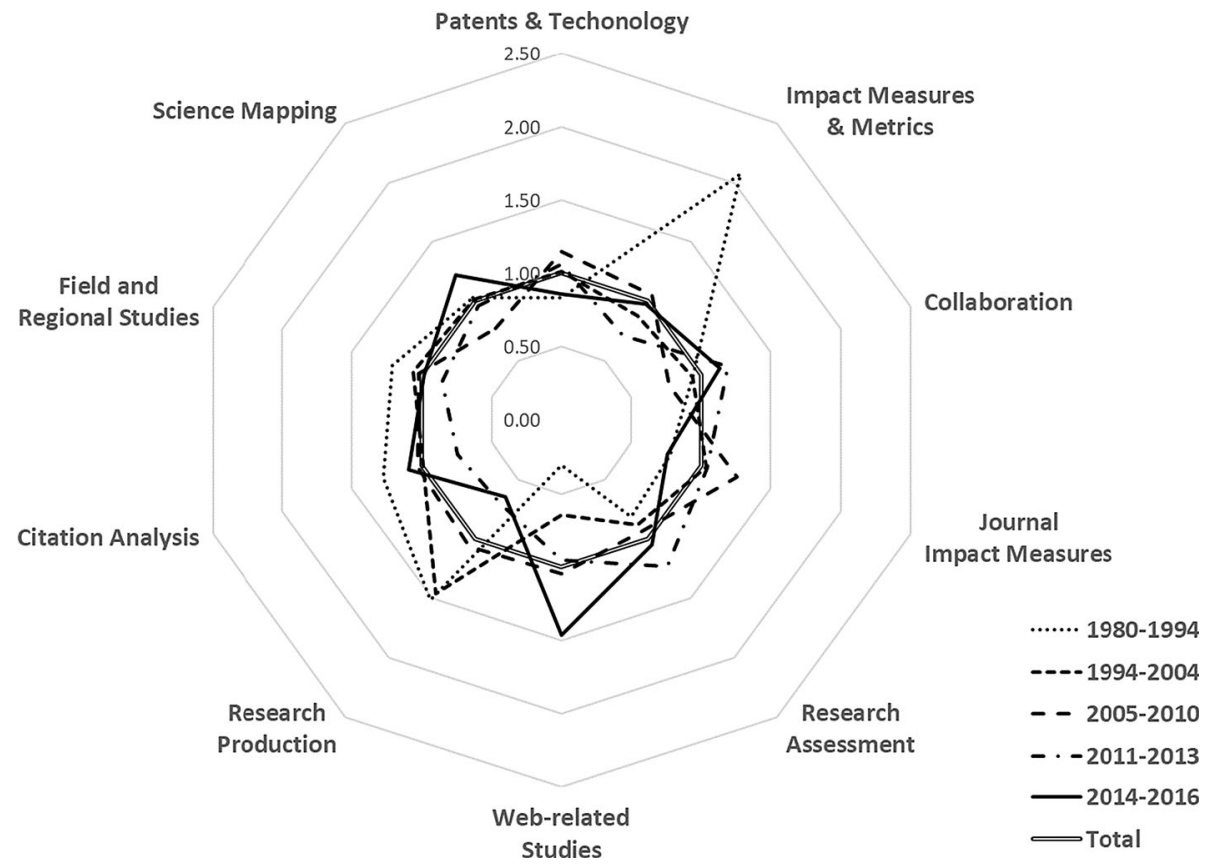

Fig. 2 Evolution of the share of 10 clusters in a lexical similarity based network of papers published in the journal Scientometrics between 1979 and 2016. Data sourced from Clarivate Analytics Web of Science Core Collection 
Table 3 Concordance ${ }^{a}$ between the clusters in the bibliographic coupling network (rows) and in the lexical based network (columns) using a cosine score. Data sourced from Clarivate Analytics Web of Science Core Collection

\begin{tabular}{|c|c|c|c|c|c|c|c|c|c|c|}
\hline & $A_{100}$ & $B_{100}$ & $C_{100}$ & $D_{100}$ & $F_{100}$ & $G_{100}$ & $H_{100}$ & $I_{100}$ & $J_{100}$ & $K_{100}$ \\
\hline$A_{0}$ & 0.60 & 0.01 & 0.06 & 0.02 & 0.07 & 0.03 & 0.03 & 0.02 & 0.04 & 0.04 \\
\hline$C_{0}$ & 0.10 & 0.17 & 0.47 & 0.09 & 0.15 & 0.05 & 0.15 & 0.06 & 0.18 & 0.06 \\
\hline$D_{0}$ & 0.07 & 0.10 & 0.05 & 0.38 & 0.16 & 0.20 & 0.11 & 0.23 & 0.14 & 0.10 \\
\hline$G_{0}$ & 0.03 & 0.01 & 0.01 & 0.05 & 0.10 & 0.08 & 0.01 & 0.01 & 0.15 & 0.05 \\
\hline$H_{0}$ & 0.10 & 0.05 & 0.05 & 0.06 & 0.11 & 0.04 & 0.26 & 0.20 & 0.10 & 0.05 \\
\hline$I_{0}$ & 0.05 & 0.06 & 0.06 & 0.12 & 0.21 & 0.15 & 0.07 & 0.15 & 0.09 & 0.04 \\
\hline$J_{0}$ & 0.03 & 0.05 & 0.04 & 0.01 & 0.27 & 0.03 & 0.05 & 0.01 & 0.14 & 0.01 \\
\hline$K_{0}$ & 0.15 & 0.04 & 0.10 & 0.04 & 0.06 & 0.05 & 0.07 & 0.09 & 0.13 & 0.52 \\
\hline
\end{tabular}

${ }^{\mathrm{a}}$ Intensity of grey shading of cells is proportional to strength of concordance

Although that cluster $D_{0}$ has a strong link with $D_{100}$, it has also strong links with the other clusters. No other cluster has so many concordance scores equal to or above 0.1 as $D_{0}$.

The objective of this paper is precisely to find out what the causes are for these transitions between the two distinct approaches. To achieve this, we present 3 intermediate, hybrid networks with different weights for both components. The properties of these approaches can be found in Table 4. We applied a 25, 50 and 75\% weight and in line with the observations in Thijs et al. (2017), we see that the introduction of some lexical information reduces the number of found clusters. With an increase of the lexical weight, the optimal number of cluster increases as well. Modularity of the detected partitioning decreases. Based on only this modularity one could argue that the bibliographic coupling approach is better, but the analysis above showed that the lexical solution is different but valid.

A more indepth view on the changes between these different approaches is provided by the alluvial chart in Fig. 3. This figure combines the five solutions with the pure bibliographic coupling in the column on the left and each step moving to the right adds $25 \%$ of weight to the lexical component to end at the right with the solution based on the pure lexical approach. The flow between each column indicates the number of documents that 
Table 4 Properties of five document networks each with different weight for the lexical component. Clusters are obtained through the Louvain method. Data sourced from Clarivate Analytics Web of Science Core Collection

\begin{tabular}{llcc}
\hline Lexical weight $(\%)$ & Density $(\%)$ & Number of clusters & Modularity \\
\hline 0 & 4.09 & 8 & 0.479 \\
25 & 3.51 & 7 & 0.449 \\
50 & 4.31 & 8 & 0.379 \\
75 & 4.60 & 9 & 0.361 \\
100 & 5.20 & 10 & 0.345 \\
\hline
\end{tabular}

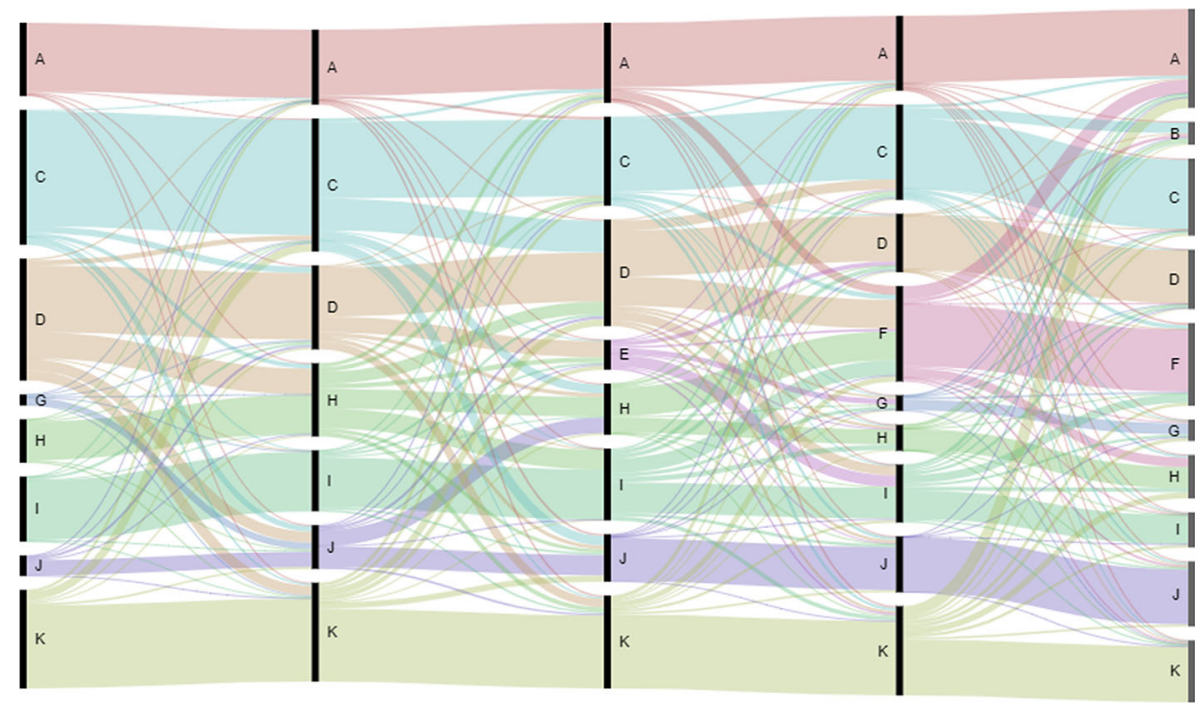

Fig. 3 Migration flow of document clusters from the journal Scientometrics in five hybrid networks combining bibliographic coupling and lexical similarity with weight for the lexical component ranging from $0 \%$ (left) over 25, 50, 75-100\% (right). Data sourced from Clarivate Analytics Web of Science Core Collection

are common between two clusters in two subsequent approaches. A document remains in the same cluster as the code of the two clusters is similar and a document is called to migrate if the codes are different. We introduce a notation for these moves between clusters but also to label a set of publications that are characterized by the same move or migration:

$$
X_{n} \rightarrow Y_{m},
$$

where $X$ and $Y$ refer to the code of the cluster and subscripts $n$ and $m$ indicate the weight of the lexical component. As we are interested in the contribution of the lexical component, we will have a premiraly look at the flow from left to right. Three main corridors can be observed in Fig. 3, which hold a strong flow of documents within the same cluster code.

$$
A_{0} \rightarrow A_{25} \rightarrow A_{50} \rightarrow A_{75} \rightarrow A_{100} \text { : Patents \& Technology }
$$


Table 5 Size and label for seven document migrations between clusters at different levels of the hybrid weight. Data sourced from Clarivate Analytics Web of Science Core Collection

\begin{tabular}{lrl}
\hline Flow & Size & Label \\
\hline$D_{0} \rightarrow J_{25}$ & 82 & Journal coverage in bibliographic databases \\
$C_{25} \rightarrow D_{50}$ & 245 & Field normalization and relative indicators \\
$H_{25} \rightarrow D_{50}$ & 186 & Aging and obsolescence of research \\
$D_{50} \rightarrow C_{75}$ & 69 & Relative citation indicators for international comparison \\
$D_{50} \rightarrow F_{75}$ & 212 & Advanced bibliometric indicators for assessment of research \\
$I_{50} \rightarrow F_{75}$ & 94 & H-index \\
$F_{75} \rightarrow A_{100}$ & 100 & Laboratories, public research institutes and funding agencies \\
\hline
\end{tabular}

$C_{0} \rightarrow C_{25} \rightarrow C_{50} \rightarrow C_{75} \rightarrow C_{100}:$ Collaboration

$K_{0} \rightarrow K_{25} \rightarrow K_{50} \rightarrow K_{75} \rightarrow K_{100}$ : Science Mapping

These corridors are also found in the concordance table between the two outer approaches.

The differences that appear in the concordance table are now clearly presented in the flow diagram. We see that cluster $J_{25}$ is nearly split in two groups, one remaining in $J$ and one migrating towards $H_{50}$. This latter deals with university rankings and with institutional name harmonisation and disambiguation. The first group relates to institutional performance in specific fields with a strong emphasis on social science. At each step cluster $D$ looses some of its members to one on the other clusters hence the many high concordance scores in Table 3.

Another interesting observation is the emergence and vanishing of cluster $E$ when moving over the $50 \%$ weight. This cluster seems to be an intermediate step between cluster $D_{25}$ and $I_{75}$. More labels for documents sets in a particular migration flow can be found in Table 5. Each of these sets holds a reasonable set of documents which cover distinct topics. Each of these topics can be linked to the particular cluster at the start and end point of the migration. None of these set are randomly grouped together.

\section{Sub-clusters}

Based on the above observations, one could formulate an alternative hypothesis stating that the changes induced by the inclusion of lexical similarities are already intrinsically present in the bibliographic-coupling network and that the addition of other components only uncovers this underlying structure. To test this hypothesis, we used two distinct approaches and selected 880 publications that were assigned to cluster $D$ in bibliographic coupling (BC) and the subset of 185 publications which migrated from $D_{0}$ towards cluster $H_{25}$ in the $25 \% \mathrm{BC}$ step. The flow of these publications can be indicated as $D_{0} \rightarrow H_{25}$.

First, we will compare the modularity score of the cluster membership of the 185 documents in the pure lexical approach with the score of the lexical partitioning in the bibliographic-coupling network. In a second step, we cluster the 880 documents in the bibliographic-coupling network and analyse some concordance between these sub-clusters and the $25 \% \mathrm{BC}$ and the pure lexical steps.

Table 6 gives the distribution of the publications that are characterised by flow $D_{0} \rightarrow H_{25}$ over clusters in the pure lexical approach. Most publications end up in cluster $H_{100}$. 40 publications can be found in $F_{100}$ after migration flow $H_{50} \rightarrow F_{75}$. Eleven 
Table 6 Distribution over clusters in pure lexical approach of 185 publications characterised by flow $D_{0} \rightarrow H_{25}$. Data sourced from Clarivate Analytics Web of Science Core Collection

\begin{tabular}{lcc}
\hline Cluster Code & Publication count & Share $(\%)$ \\
\hline$A_{100}$ & 18 & 9.7 \\
$B_{100}$ & 7 & 3.8 \\
$C_{100}$ & 8 & 4.3 \\
$D_{100}$ & 10 & 5.4 \\
$F_{100}$ & 40 & 21.6 \\
$G_{100}$ & 9 & 4.9 \\
$H_{100}$ & 46 & 24.9 \\
$I_{100}$ & 21 & 11.4 \\
$J_{100}$ & 11 & 5.9 \\
$K_{100}$ & 4 & 2.2 \\
Not clustered & 11 & 5.9 \\
\hline
\end{tabular}

publications, that had a cluster assignment in the first two steps, were excluded from the pure lexical analysis as they did not belong to the largest component in that network.

Using the partitioning presented in Table 6 in a pure lexical based network of these 174 documents results in a modularity of 0.32 while the same groups in a bibliographic coupling network results in a modularity score of 0.15 . Although modularity scores are often incomparable (Good et al. 2010), they can be used for comparison of network of equal size and similar cluster distributions. Closer inspection reveals that publications in flow $D_{0} \rightarrow H_{25} \rightarrow H_{100}$ are strongly focusing on gender differences in research while $D_{0} \rightarrow H_{25} \rightarrow F_{100}$ papers deal with delayed recognition and 'Sleeping Beauties'. The link to gender-research in Scientometrics is clearly present in the other smaller clusters. $D_{0} \rightarrow H_{25} \rightarrow C_{100}$ papers study the role of female researchers in collaboration, in $D_{0} \rightarrow H_{25} \rightarrow H_{100}$ most papers are gender studies but use a publication set retrieved from Google Scholar.

For the second test we ran the Louvain Community detection algorithm again on the 880 documents in cluster $D_{0}$. This resulted in 11 clusters (see Table 7). Discussing these eleven sub-clusters would take us too far but the more interesting analysis regarding the concordance between the sub-clusters from Table 7 and the partitioning of the 185 $D_{0} \rightarrow H_{25}$ papers as presented in Table 6 .

We see that nearly all documents that migrate from $D_{0}$ to $H_{25}$ are located in sub-clusters 5 and 6 in Table 8 . These sub-clusters take $84.3 \%$ of all papers in this flow and $77 \%$ of the papers assigned to these two sub-clusters follow this flow. This indicates that the flow caused by adding a small portion of lexical information amplifies the substructure hidden in the bibliographic-coupling network. Sub-cluster 5 relates to highly cited papers and the Matthew effect as opposed to delayed recognition while sub-cluster 6 includes papers on the relation between science production and personal and sociographical properties of mainly authors. This sub-cluster has a subset of papers on gender but has much broader scope.

The scattered profile of the publications from the sub-clusters over distinct clusters in the lexical approach proves that the difference between the two approaches. As it is possible to delineate the topic of papers moving along in the same flow it is clear that these migrations are not just random but are valid identifiers of underlying topics hidden in the lexical structure of the document network and not present in the citation-link based one. 
Table 7 Distribution over sub-clusters of 880 publications from cluster $D$ in the bibliographic-coupling network. Data sourced from Clarivate Analytics Web of Science Core Collection

\begin{tabular}{|c|c|c|c|c|c|c|c|c|c|c|c|}
\hline Sub-cluster number & 1 & 2 & 3 & 4 & 5 & 6 & 7 & 8 & 9 & 10 & 11 \\
\hline Publication count & 88 & 137 & 54 & 209 & 104 & 99 & 45 & 21 & 97 & 17 & 9 \\
\hline
\end{tabular}

Table 8 Distribution of 185 publications from flow $D_{0} \rightarrow H_{25}$ in sub-clusters 5 and 6 over clusters from the lexical approach. Data sourced from Clarivate Analytics Web of Science Core Collection

\begin{tabular}{lrrrrrrrrrrrr}
\hline Sub-cluster & $A_{100}$ & $B_{100}$ & $C_{100}$ & $D_{100}$ & $F_{100}$ & $G_{100}$ & $H_{100}$ & $I_{100}$ & $J_{100}$ & $K_{100}$ & NC & Total \\
\hline 5 & 7 & 2 & 5 & 4 & 27 & 5 & 25 & 4 & 4 & & 1 & 84 \\
6 & 8 & 5 & 3 & 6 & 5 & 2 & 11 & 13 & 5 & 4 & 10 & 72 \\
Publication count & 18 & 7 & 8 & 10 & 40 & 9 & 46 & 21 & 11 & 4 & 11 & 185 \\
\hline
\end{tabular}

\section{Discussion and conclusions}

The four decades of publications in Scientometrics provide us a unique opportunity for the closer inspection of the added value of the lexical document similarities in science mapping exercises. The structure of the field has been studied before and our own knowledge of the field allows us to validate the outcome of the topic detection analyses without consulting external experts. The qualitative analysis of the cluster structure of both a pure linkbased and a lexical similarity based document network proved the validity of both approaches. Despite the lower modularity score for the text based network there is no indication that this network is of lesser quality then the one based bibliographic coupling. Earlier research already showed that a hybrid approach with a small weight on the lexical component improves the bibliographic-coupling based partitioning. But a more detailed and closer inspection of the stepwise contribution of the lexical component revealed that much smaller but homogeneous topics are migrating from one cluster to another. Moreover, these smaller groups of papers are not hidden in the link-based network but emerge once lexical information is added. Sub-clusters could not explain the distribution over distinct topics.

When looking at the migrating topics it is striking that most of them are related to both larger clusters. Some of the papers even exhibit two or more migration steps and can be linked to up to three different topics. Such a fine-grained identification of topics and their relatedness leads automatically to other questions related to the identification of topics. First, one question relates to the definition of a topic, should this be restricted to single concepts and should we incorporate in each cluster exercise the possibility of multiple topic assignments of document or can topics be linked to multiple concepts as we see in this analysis which have to be detected after the clustering. Next, too many small topics might hinder a clear cognitive understanding of the field structure but, given the validity of their existence, it also seems not correct to ignore them. This stresses the importance of the delineation of the objectives for the clustering exercise as this will be the only reference for the decision on the level of analysis. 


\section{References}

Blondel, V. D., Guillaume, J. L., Lambiotte, R., \& Lefebvre, E. (2008). Fast unfolding of communities in large networks. Journal of Statistical Mechanics: Theory and Experiment, 10, P10008.

Boyack, K. W., \& Klavans, R. (2010). Co-citation analysis, bibliographic coupling, and direct citation: Which citation approach represents the research front most accurately? Journal of the American Society for Information Science and Technology, 61(12), 2389-2404.

Callon, M., Courtial, J. P., \& Laville, F. (1991). Co-word analysis as a tool for describing the network of interactions between basic and technological research: The case of polymer chemistry. Scientometrics, 22(1), 155-205.

Garfield, E. (1969). Permuterm Subject Index-The primordial dictionary of science. Current Contents, $12(22), 4$.

Glänzel, W., \& Thijs, B. (2011). Using 'core documents' for the representation of clusters and topics. Scientometrics, 88(1), 297-309.

Glänzel, W., \& Thijs, B. (2012). Hybrid solutions-The best of all possible worlds? Bibliometrie \& Praxis und Forschung, 1(3), URN:urn:nbn:de:bvb:355-152-4.

Glänzel, W., \& Thijs, B. (2017). Using hybrid methods and core documents for the representation of clusters and topics. The astronomy dataset. Scientometrics, 111(2), 1071-1087.

Glenisson, P., Glänzel, W., Janssens, F., \& de Moor, B. (2005). Combining full text and bibliometric information in mapping scientific disciplines. Information Processing and Management, 41(6), $1548-1572$.

Good, B. H., de Montojoye, Y.-A., \& Clauset, A. (2010). Performance of modularity maximization in practical contexts. Physical Review E, 81, 046106.

Janssens, F., Glänzel, W., \& de Moor, B. (2008). A hybrid mapping of information science. Scientometrics, 75(3), 607-631.

Manning, Ch. D., Surdeanu, M., Bauer, J., Finkel, J., Bethard, S. J., \& McClosky, D. (2014). The Stanford CoreNLP Natural Language Processing Toolkit. In Proceedings of the 52nd annual meeting of the association for computational linguistics: system demonstrations (pp. 55-60).

Thijs, B., Glänzel, W., \& Meyer, M. (2017). Improved lexical similarities for hybrid clustering through the use of noun phrases extraction. FEB Research Report MSI_1703, MSI_1703. Leuven (Belgium): KU Leuven, Faculty of Economics and Business.

Todorov, R., \& Winterhager, M. (1990). Mapping Australian geophysics-A co-heading analysis. Scientometrics, 19(1-2), 35-56. 